\title{
Phonocardiographic manifestations of heterograft valve dysfunction in the mitral area
}

\author{
Barry Stimmel, Elliott Stein, Arnold M. Katz, Robert S. Litwak, and \\ Ephraim Donoso \\ From the Department of Medicine, Division of Cardiology, and the Department of Surgery, \\ Division of Cardiothoracic Surgery, of the Mount Sinai School of Medicine, \\ City University of New York, New York, U.S.A.
}

Phonocardiographic and pathological features of mitral porcine aortic heterograft valve failure are described in 10 patients with rheumatic valvular disease. Six had isolated mitral porcine aortic heterograft valve; 3 also had one in the aortic area; one had this in both mitral and tricuspid areas with an aortic ball valve prosthesis. All patients had systolic murmurs at the apex indicative of mitral insufficiency, 8 had prominent third heart sounds. Five had specific and unusual variations in the systolic murmurs consisting of $(I)$ conspicuous alternation of systolic murmur intensity with heart beat independent of preceding cycle length and/or (2) division of the systolic murmur into distinct components. Seven required reoperation with replacement of the mitral porcine aortic heterograft valve. Commissural separation from the stent was the major finding in 4; primary leaflet damage in 3. Only patients with commissural separation from the stent exhibited these two specific and unusual variations of the systolic murmur which thus appear to result from oscillation of the torn commissure. A systolic murmur consisting of several distinct components and/or varying intensity may therefore be presumptive evidence for commissural separation from the stent of the heterograft valve.

During the past several years, porcine aortic heterograft valves have been employed at various centres as prosthetic devices in patients undergoing mitral valve replacement (Carpentier et al., 1969; Kouchoukos et al., 1969; Litwak et al., I97I). In this institution porcine aortic heterograft valves were used in selected patients during 1969 and 1970.

Review of phonocardiograms taken in several of these patients revealed unique findings not present in other forms of mitral regurgitation. The purpose of this paper is to report the distinctive phonocardiographic findings and to attempt to correlate these results with pathological examination of defective valves.

\section{Methods}

All ro of the patients studied had rheumatic heart disease. Six patients had primarily mitral valve disease and received a heterograft in the mitral area. Three patients had mitral and aortic valve disease and received heterografts in both the mitral and aortic areas. One patient had disease of the

Received 2I January 1972. aortic, mitral, and tricuspid valve and received heterografts in the mitral valve area and the tricuspid valve area with a Starr Edwards ball valve prosthesis in the aortic area. During operation all mitral infravalvular structures were removed. The heterograft valves used were all formalin fixed porcine aortic valves sewn onto a high porosity Dacron cloth-covered stent machined from a solid piece of stellite No. 25 . Details of the valve preparation and mounting have been previously described (Litwak et al., 1971). Seven of these patients have required reoperation with replacement of the defective heterograft valve with ball valve prosthesis. Three patients, one with a double heterograft, in spite of clinically shown valve malfunction are not sufficiently disabled at the present time to warrant corrective surgery. All of the reoperated patients had phonocardiograms immediately before operation. The 3 remaining patients had phonocardiograms on an ambulatory basis. The phonocardiograms were performed on an Electronics for Medicine DR8 recorder at a paper speed of $75 \mathrm{~mm}$ per second, using crystal microphones, and a standard pulse pick-up for carotid pulse, jugular venous pulse, and apex motion. Pass ban filters of low, medium, medium to high, and high frequency types were employed in the sound recordings. 
All recordings were made with the breath held in full expiration. Measurement of time intervals wwas made from the onset of the $Q$ wave of the electrocardiogram to the onset of the major de-

- flections of the heart sounds or from the onset of the aortic component of the second sound to the onset of the third heart sound. Time intervals corrected for heart rate were measured in 5 consecutive cycle lengths with the mean values reported.

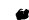

\section{Results}

Six of the 7 patients with heterografts in 4 mitral valve area were in atrial fibrillation; one had a right bundle-branch block. All 3 patients with heterografts in both aortic and mitral areas were in normal sinus rhythm.

The first sound was recorded without diffi-

b culty in all patients. In 2 out of 7 patients with mitral valve heterografts and one of the 3

- patients with aortic and mitral heterografts, a low frequency muscular component was identified 0.03 to $0.04 \mathrm{sec}$ after the $Q$ wave on the electrocardiogram. Two components of the first sound were identified in 3 of the 7 patients with mitral heterografts and $I$ of the 3 patients with heterografts in both areas. The first component (Q-I) appeared between 0.06 to $0.08 \mathrm{sec}$ and the second component (Q-2), when present, appeared $0.08-0.10 \mathrm{sec}$ after the $Q$ wave of the electrocardiogram. A consistent variation in intensity with the first heart sound as related to previous cycle length could not be shown; in some instances the intensity of SI decreased and in some instances

it increased after long cycle lengths.

An ejection sound was recorded in 2 of the 7 patients with mitral valve heterografts and 2 out of the 3 patients with heterografts in both valve areas. This sound appeared 0.12 4j $0.17 \mathrm{sec}$ after the $Q$ wave of the electrocardiogram and may have been related to pul- monary hypertension.

The aortic and pulmonary components of * the second sound were identified in all patients. There were no significant abnormalities of this sound.

- A third heart sound was seen in 6 of the 7 patients with mitral valve heterografts and 2

- of the 3 patients with heterografts in both areas. The interval between the aortic com-

- ponent of the second sound and the third sound $\left(\mathrm{S}_{3}\right)\left(\mathrm{A}_{2}-\mathrm{S}_{3}\right)$ varied from 0.12 to 0.18 sec.

* All the patients had medium-high frequency, high intensity systolic murmurs

1 starting with the second component of the first sound and ending shortly before the aortic component of the second sound. Two distinctive features were noted in the systolic

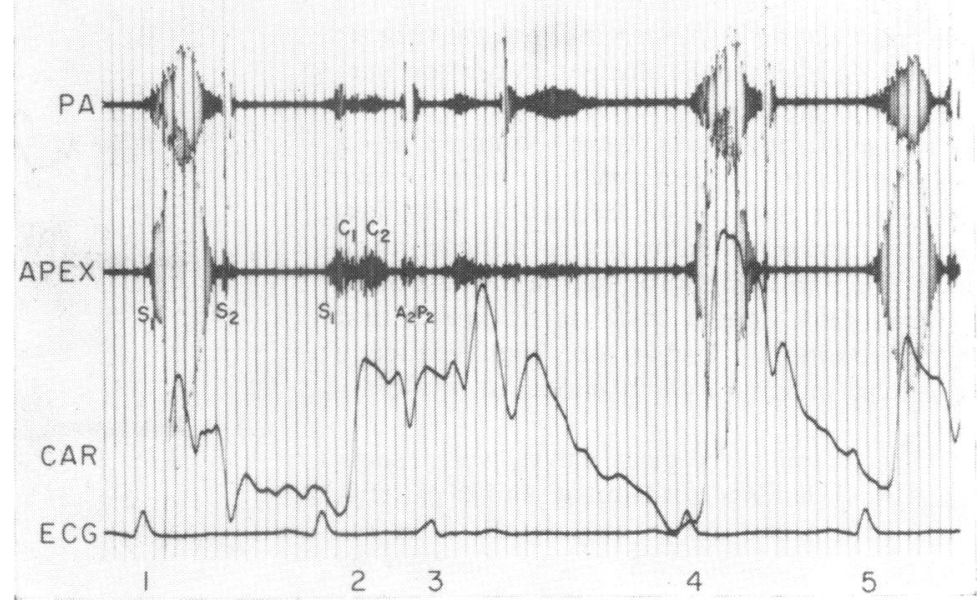

FIG. I The phonocardiogram recorded from the apical and pulmonary $(P A)$ area in Case 9. Normal sinus rhythm is present with a premature ventricular contraction in cycle 3. $A$ significant difference in intensity exists between cycle 1,2 , and 5. Division of the murmur into components is also present $\left(C_{I}, C_{2}\right)$.

murmur: a variation of intensity and a division of the murmur into components. Four out of 7 patients with mitral valve heterografts and 2 of the 3 patients with heterografts in both valve areas had a conspicuous alteration in intensity of the murmur not related to preceding cycle length in the patients with atrial fibrillation and present in patients with normal sinus rhythm (Fig. I and 2). Three patients with mitral valve heterografts and 2 patients with mitral and aortic heterografts were also

FIG. 2 The phonocardiogram recorded from the apical area in Case 6. Atrial fibrillation is present. An alternation in intensity and duration of murmur is seen between cycles 1,2 , and 4. $A$ short diastolic murmur (DM) is also present.

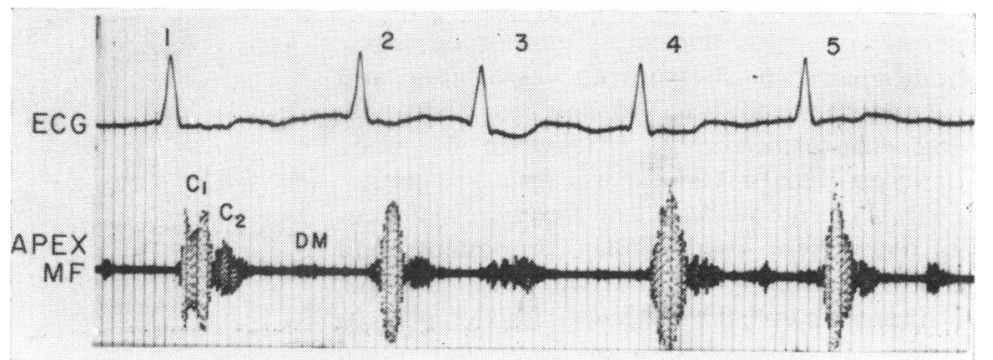


noted to have 2 or 3 distinct components to the murmur giving it a 'warbling' quality (Fig. I, 3, and 4). Neither the alternation nor the warbling was affected by respiration.

Diastolic murmurs were observed in 2 patients. One patient with a mitral valve heterograft had a low frequency mid-diastolic murmur, probably representing a mitral flow murmur and ending before the first heart sound. One patient with aortic and mitral valve heterografts had an early high frequency diastolic murmur which ended in late diastole.

The mitral heterograft valves were examined in 5 patients with mitral and 2 patients with mitral and aortic heterografts after reoperatior. Abnormalities were classified as (I) leaflet, (2) leaflet separation from the stent at the commissure and, (3) stent (Table 2). Two of the 5 patients with mitral heterografts and both patients with both heterografts exhibited commissural separation. Four of 5 patients with mitral heterografts had leaflet abnormalities ranging from minor alterations such as sagging of the leaflets to inversion and, in one instance, perforation of the cusp. No abnormalities of the stent were noted. Correlation of the pathological with the phonocardiographic findings revealed the alternation or warbling of the systolic murmur to be present in all cases of commissural separation (Table 3). In the 2 instances with only leaflet damage, the distinctive findings in the systolic murmur were not seen. Of the 3 unoperated patients, alternation was seen in one.

\section{Discussion}

All patients in this study had mitral insufficiency secondary to failure of the porcine heterograft. Though 3 of the patients had initial replacement of the aortic valve with a heterograft as well as the mitral valve, and one patient had a Starr Edwards aortic prosthesis, at the time of the study their aortic valves were functioning normally and, for purposes of discussion, all patients will be considered in one group. Gianelly, Popp, and Hultgren (1970) reported phonocardiographic findings in patients with normally functioning aortic homografts in the mitral valve area and related these findings to angiocardiographic and echocardiographic studies on the same patients. The first sound was found to consist of four components: a low frequency muscular component, two valvular components, and a late ejection sound that was seen in some, but not all, of the patients. In the present study, the existence of a low frequency sound

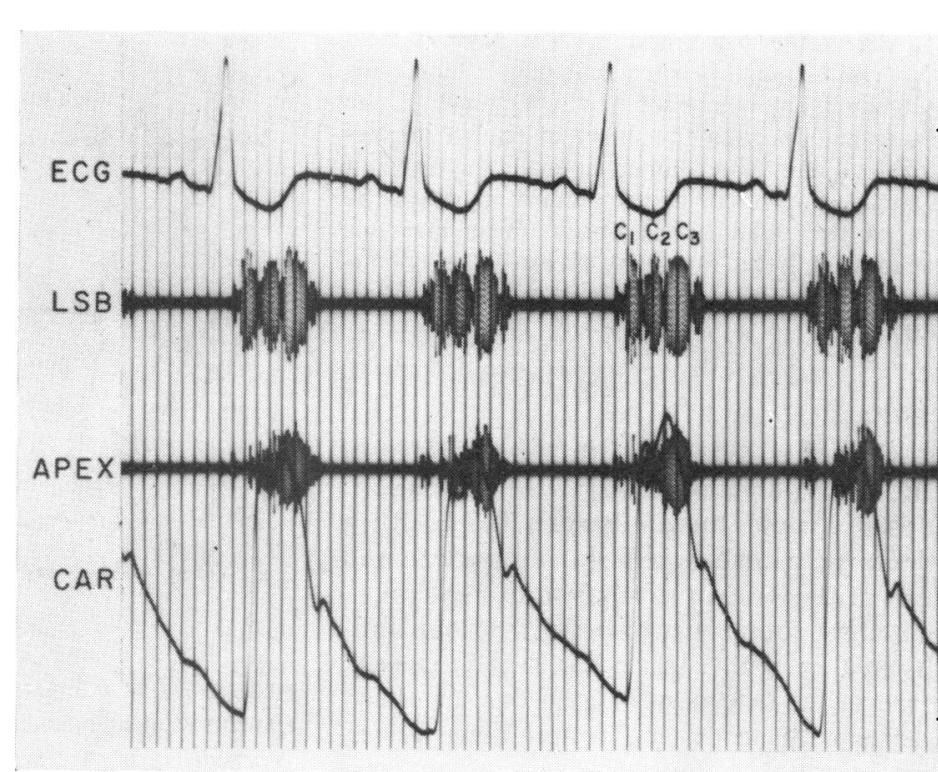

FIG. 3 The phonocardiogram recorded from the apex and left sternal border (LSB) in Case 10. Normal sinus rhythm is present. The murmur can be separated into three distinct components $\left(C_{1}, C_{2}, C_{3}\right)$.

FIG. 4 The phonocardiogram recorded from apex and left sternal border in Case 2. Atrial fibrillation with a right bundle-branch block is present. Division of the murmur into two components is seen as well as abrupt cessation of the murmur $0.08 \mathrm{sec}$ before $S_{2}$ in cycle 2.

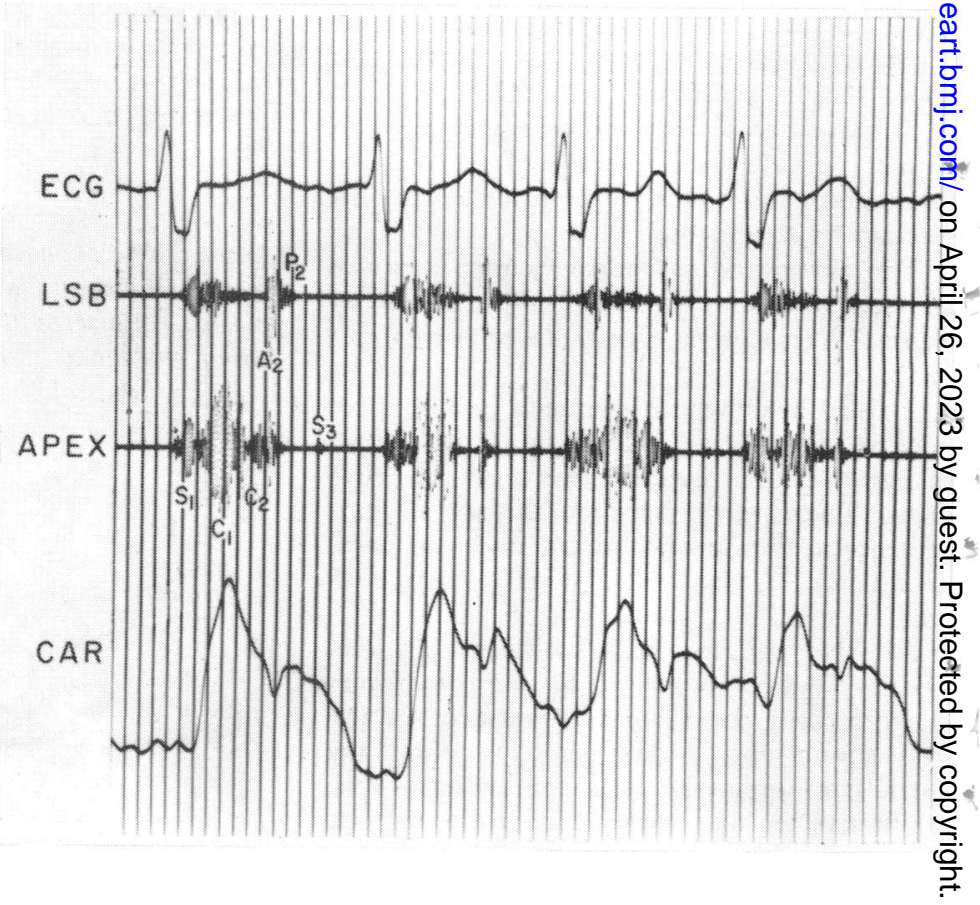


TABLE I Phonocardiographic findings: heart sounds

\begin{tabular}{|c|c|c|c|c|c|c|c|c|c|}
\hline $\begin{array}{l}\text { Case } \\
\text { No. }\end{array}$ & Rhythm & Valve & $Q R S$ & $Q-M$ & $Q-I$ & $Q-2$ & $Q-E S$ & $A_{2} P_{2}$ & $A_{2} S_{3}$ \\
\hline I & Atrial fibrillation & MVH & 0.08 & - & 0.07 & 0.10 & - & 0.02 & 0.16 \\
\hline 2 & Atrial fibrillation & MVH/TVH/SEA & 0.12 & 0.04 & 0.07 & - & - & 0.04 & 0.13 \\
\hline 3 & Atrial fibrillation & MVH & 0.09 & - & 0.06 & - & - & 0.04 & 0.15 \\
\hline 4 & Atrial fibrillation & MVH & 0.09 & - & 0.08 & $0 \cdot 10$ & - & 0.03 & 0.12 \\
\hline 5 & Atrial fibrillation & MVH & 0.07 & 0.04 & 0.08 & 0.10 & 0.17 & 0.02 & 0.18 \\
\hline 6 & Atrial fibrillation & MVH & $0 \cdot 10$ & - & 0.07 & - & - & 0.02 & 0.17 \\
\hline-7 & $\begin{array}{l}\text { Normal sinus rhythm/ } \\
\text { intraventricular conduc- } \\
\text { tion defect }\end{array}$ & MVH & $0 \cdot 10$ & - & 0.08 & - & $0 \cdot 12$ & 0.05 & 0.15 \\
\hline 8 & Normal sinus rhythm & BVH & 0.09 & 0.03 & 0.06 & 0.10 & 0.12 & 0.03 & - \\
\hline 9 & Normal sinus rhythm & BVH & 0.08 & - & 0.08 & - & - & 0.02 & 0.12 \\
\hline 410 & Normal sinus rhythm & $\mathrm{BVH}$ & 0.09 & - & 0.06 & - & 0.14 & 0.02 & 0.15 \\
\hline
\end{tabular}

Abbreviations: $Q-M=Q$ wave of electrocardiogram to onset of initial low frequency component before first sound; $Q-I=Q$ to onset of first loud component of first sound; $Q-2=Q$ to onset second loud component first sound; Q-ES $=Q$ to ejection sound; $A_{2} P_{2}=$ distance between aortic and pulmonic components of second sound; $A_{2} S_{3}=$ component of second sound to third heart sound; $M V H=$ heterograft in mitral area; SEA = Starr Edwards Ball i valve aortic area; $\mathrm{TVH}=$ heterograft in tricuspid area; $\mathrm{BVH}=$ heterografts in both aortic and mitral areas.

after the QRS was seen in only 3 of the ro cases. The reason for this discrepancy may be related to a difference in recording technique.

The valvular components of the first sound . were shown by Gianelly et al. (1970) to consist of an initial sound occurring when the searly systolic ballooning of the graft leaflets was suddenly checked. The second component occurred at the time when the leaflets were suddenly jerked back toward the base of the heart at the onset of ventricular ejection. - The absence of the second component in 6 of Io patients in our series might be related to either technique or to the presence of significant mitral regurgitation which obscured this sound. An alternative explanation is that the conspicuous insufficiency prevents the 'sudden acceleration of a mass of blood behind tensed valve leaflets' which has been proposed as the mechanism of this sound. A sound occurring 0.12 to $0.17 \mathrm{sec}$ after the $Q$ wave was seen in 4 out of 10 patients. In 2 of these patients, coexisting aortic valve heterografts were present and the ejection sound might have been related to some abnormalities of the aortic ring or alternately pulmonary hypertension.

A third heart sound was noted in 9 out of the ro patients. Two current theories exist as to the origin of the third heart sound. The most common relates the sound to the rapid increase in left ventricular filling which causes the ventricular musculature to check itself suddenly when distended, producing a vibration (Potain, 1900; Crevasse et al., 1962). Many investigators, however, feel the third

TABLE 2 Phonocardiographic and pathological findings

\begin{tabular}{|c|c|c|c|c|c|c|c|c|}
\hline \multirow{2}{*}{$\begin{array}{l}\text { Case } \\
\text { No. }\end{array}$} & \multicolumn{3}{|c|}{ Systolic murmur } & \multirow[t]{2}{*}{ Diastolic } & \multirow[t]{2}{*}{ Apex cardiogram } & \multicolumn{3}{|c|}{ Abnormality of heterograft } \\
\hline & Present & $\begin{array}{l}\text { Alterna- } \\
\text { tion }\end{array}$ & $\begin{array}{l}\text { Compo- } \\
\text { nents }\end{array}$ & & & Leaflet & $\begin{array}{l}\text { Separation } \\
\text { from stent }\end{array}$ & Stent \\
\hline$\sim$ & + & + & + & o & Normal & Sagging & $+/$ calcification & Normal \\
\hline 2 & + & + & + & + & $\begin{array}{l}\text { Prominent rapid } \\
\text { filling wave }\end{array}$ & Inversion & $+/$ calcification & Normal \\
\hline 3 & + & 0 & 0 & 0 & $\begin{array}{l}\text { Prominent rapid } \\
\text { filling wave }\end{array}$ & Contraction & Normal & Normal \\
\hline 4 & + & + & $\circ$ & & Normal & - & - & 一 \\
\hline 5 & + & $\circ$ & $\circ$ & ० & Normal & - & - & - \\
\hline 6 & + & + & + & ० & Normal & Normal & + & Normal \\
\hline 7 & + & $\circ$ & $\circ$ & $\circ$ & $\begin{array}{l}\text { Prominent rapid } \\
\text { filling wave }\end{array}$ & $\begin{array}{c}\text { Perforation } \\
\text { of leaflet }\end{array}$ & Normal & Normal \\
\hline & + & 0 & o & + & $\begin{array}{l}\text { Prominent systolic } \\
\text { wave }\end{array}$ & 一 & - & - \\
\hline 9 & + & + & + & o & Systolic plateau & Normal & + & Normal \\
\hline IO & + & + & + & 0 & Normal & Normal & + & Normal \\
\hline
\end{tabular}

Abbreviations: $+=$ present $; 0=$ absent $;-=$ not known. 
TABLE 3 Correlation of changes in the systolic murmur with pathological findings at operation

\begin{tabular}{llll}
\hline & Unknown & $\begin{array}{l}\text { Leaflet } \\
\text { (only) }\end{array}$ & Commissure \\
\hline Holosystolic & & & \\
$\quad$ murmur only & 3 & 2 & 0 \\
Alternation & 1 & 0 & 5 \\
Components & 0 & 0 & 5 \\
\hline
\end{tabular}

sound is subvalvular in origin and represents tension of chordae or papillary muscles during early diastole at the end of the rapid filling phase (Nixon, 1961 ; Fleming, 1969).

Evidence to support the subvalvular origin of this sound has come from Fleming (1969) who noted the absence of a third sound in patients with ball valve prosthesis. However, third heart sounds have been recorded recently in patients with both normally functioning and failing heterografts (Gianelly $e t$ al., 1970; El Gamal and Smith, 1970). Since patients with heterograft replacements have the chordae and papillary muscles removed, it is likely that other factors such as left ventricular wall motion may play a significant role in the production of the third sound.

The most interesting findings are the alternation in intensity and the warbling quality of the systolic murmur seen in the patients with commissural separation from the stent. Pathological examination of heterograft failures has shown several types of abnormalities, the most common being commissural separation from the stent. However, leaflet perforation, prolapse, or sagging of the leaflets, and peripheral leak around the sewing ring have also been described. Separation of the leaflet from the stent was seen in 5 of the 7 cases examined, being the sole abnormality found in 3. Leaflet abnormalities were found in 4 of the patients, in 2 coexisting with separation of the commissure from the stent.

All those patients manifesting separation of the commissure exhibited changes in alternation and division of the systolic murmur. The mechanism of the production of this unique murmur is unclear. However, it is possible, using the pathological findings, to hypothesize the aetiology. The separation from the stent at the commissural area might result in a random variation of valve leaflet position at the beginning of each cardiac contraction consequently affecting the degree of regurgitant flow and the intensity of the murmur. Another possibility is that the murmur results from two different sources, one the turbulence of the regurgitant blood and the second the vibrations of the non-attached commissure. The random variation of commissure position at the beginning of each contraction may determine the degree and type of vibration for that particular cycle. The amount of regurgitant flow need not change if the second explanation is correct.

Though 6 of the patients studied were in atrial fibrillation, which would predispose to a varying volume of blood ejected with each cardiac cycle, this finding was present also in patients in normal sinus rhythm as well as being absent in those patients with atrial fibrillation and primarily leaflet abnormalities. It seems, therefore, that the intensity of the murmur depended not as much on the cycle length as on the varying position of the separated commissures.

This variation in leaflet position might also result in a division of the murmur into components secondary to the oscillation of the free commissural edge with each cardiac contraction.

In order to delineate further the origin of these changes, correlation of angiographic and phonographic studies will be carried out in the future. It should be noted that Killebrew and Cohn (1971) described unusual murmurs in patients with heterograft mitral valve failures. These murmurs were described as having a 'honking' quality and were associated with abnormalities of the valve leaflets. One patient, however, had a 'machine-gun' type murmur which had several distinct components similar to those found in the present study. At operation, this patient had a separation of his leaflet from the stent at the commissural area. This finding serves to corroborate the association made between the changes in the systolic murmur and the pathological findings of the heterograft valve abnormalities.

It is suggested that a patient with a mitral valve heterograft who has a holosystolic murmur, alternating in intensity from beat to beat and consisting of several components, may have an insufficiency due to commissural separation of the valve leaflets from the stent.

\section{References}

Carpentier, A., Lemaigre, G., Robert, L., Carpentier, S., and Dubost, C. (1969). Biological factors affecting long-term results of valvular heterografts. Fournal of Thoracic and Cardiovascular Surgery, 58, 467.

Crevasse, L., Wheat, M. W., Wilson, J. R., Leeds, R. F., and Taylor, W. J. (1962). The mechanism of the generation of the third and fourth heart sounds. Circulation, 25, 635 . 
El Gamal, M., and Smith, D. R. (1970). Occurrence of a left ventricular third heart sound in incom- petent mitral heterografts. British Heart fournal, 32, 497 .

Fleming, J. S. (1969). Evidence for a mitral valve origin of the left ventricular third heart sound. British Heart fournal, 31, 192.

Gianelly, R. E., Popp, R. L., and Hultgren, H. N. (1970). Heart sounds in patients with homograft replacement of the mitral valve. Circulation, 42, 309.

Killebrew, E., and Cohn, K. (197I). Observations on

- murmurs originating from incompetent heterograft mitral valves. American Heart fournal, 81, 490.

Kouchoukos, N. T., Kerr, A. R., Sheppard, L. C., Ceballase, R., and Kirklin, J. W. (I969). Hetero- graft replacement of the mitral valve. Circulation, 39-40, Suppl. III, I25.

Litwak, R. S., Hancock, W. B., Lukban, S. B., Jurado, R. A., and Olsen, E. G. J. (I97I). Results of mitral valve replacement with formalin fixed porcine xenografts. Presented at American College of Cardiology Meeting, Jan. 10-12, I971.

Nixon, P. G. F. (I96I). The third heart sound in mitral regurgitation. British Heart fournal, 23, 677.

Potain, P. C. E. (1900). Les bruits de galop. Semaine Médicale, 20, 175 .

Requests for reprints to Dr. Barry Stimmel, Mount Sinai School of Medicine, I2I2 Fifth Avenue, New York, N.Y. I0029, U.S.A. 